\title{
Exploring chemo-mechanics of granular material using DEM
}

\author{
P. Viswanath ${ }^{1}$, and Arghya Das $^{2 *}$ \\ ${ }^{1}$ Research Scholar, Civil Engineering Department, IIT Kanpur-208016, India \\ ${ }^{2}$ Assistant Professor, Civil Engineering Department, IIT Kanpur-208016, India
}

\begin{abstract}
Particle Size Distribution (PSD) is one of the prime guiding factors of granular media response. Degradation via weathering is a process, which brings about a gradual shift in the PSD. In nature, chemically sensitive material like calcite undergoes chemo-mechanical degradation bringing about variations in their behaviour. In the present study, an experimental investigation is carried out to get insight into the mechanical response during the coupled chemo-mechanical process. The experiments were carried out at two different rates of dissolutions in a custom made 1D compression mould. From the experiments, it is clear that the higher rate of dissolution reduces the lateral earth pressure more than the lower rate. Discrete Element Method (DEM) analyses the micromechanical process behind the observed response from experiments. The results showed a reduction in lateral stress as soon as the dissolution starts. DEM analysis confirms the competing mechanism between grain size reduction and grain rearrangement as the guiding element for the granular media response.
\end{abstract}

\section{Introduction}

PSD controls both volumetric [1-2] and the stress response [3] of granular materials. PSD variation under mechanical load alone has been in focus through grain breakage studies [4-6]. Another factor for PSD variation is the dissolution of particles. Dissolution of bonded geomaterial under Chemo-mechanical loading shows softening of the material [7]. The impact of such chemomechanics on pure granular material is somewhat less explored [8]. Researches have shown a reduction of lateral stress, and a subsequent regain of the same for a mixture of inert geomaterial with an active salt under mechanical loading under the continuous dissolution of salts [9-10]. Thus the chemo-mechanics of pure granular material needs further investigation. Though the experimental studies explore the macromechanics of the process, DEM provides the micromechanics behind the same [11-12]. Thus in this paper, the chemo-mechanics of granular material is explored in detail with the help of DEM.

\section{Experimental investigation}

\subsection{Material property}

The material under the present study is a commercially available calcite granule found in India. It has 95\% calcium carbonate in its mineral composition. The PSD is uniform and has a minimum and maximum void ratios of 0.722 and 0.818 , respectively [13]. The particles are angular and with a specific gravity of 2.65. These particles are prone to dissolution by mild acid, and thus for the study, acetic acid is used as a solvent.

\subsection{Test methodology}

The experiments are carried out on a custom made 1D compression setup taking ideas from the 'soft oedometer' [14] to measure hoop strain. The samples of $50 \%$ relative density were placed inside the setup, instrumented with measuring strain continuously. The setup is connected to the flow channels with a suction pressure applied by a peristaltic pump at the top. Thus, the flow is always upward, enhancing the escape of carbon dioxide formed during dissolution. The flow rate variation during the whole experiment for three repeated tests is shown in figure 1.

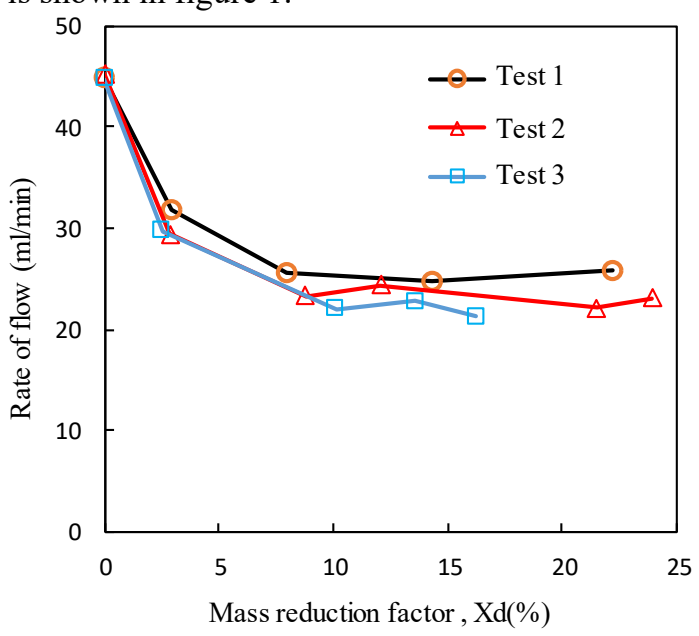

Fig. 1. Flow variation with a mass reduction factor.

\footnotetext{
* Corresponding author: arghya@iitk.ac.in

A video is available at https://doi.org/10.48448/kfwq-wq15
} 
Experiments were carried out at two different $\mathrm{pH}$ (2.8 \&3.3), to explore the effects of the rate of dissolution on the mechanical response. The samples are collected in the outlet after dissolution for the ion analysis to calculate the mass dissolution. In order to quantify the degree of dissolution at different stages, a mass reduction factor $\left(\chi_{d}\right)$ is utilized. It is the ratio of mass dissolved upon original mass. The desired axial stress of $580 \mathrm{kPa}$ was reached by incremental loading. After reaching the desired axial stress, the sample is initially flushed with deionized water to flush out air. Then the desired $\mathrm{pH}$ solution is allowed to pass continuously with the strain gauge reading being monitored simultaneously. The detailed layout of the whole system is shown in figure 2 . The experiments were stopped once the axial strain is around $10 \%$.

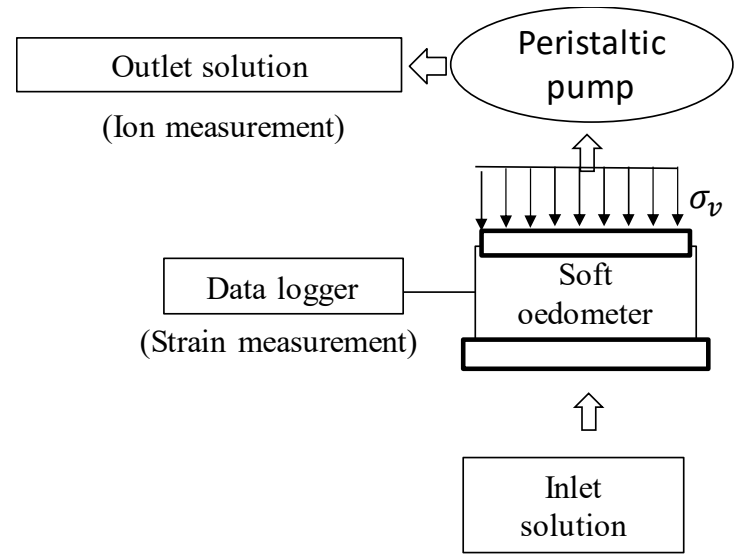

Fig. 2. The layout of the test setup.

\subsection{Experimental observations}

As the dissolution triggered, a reduction in earth pressure coefficient (EPC) (EPC is the ratio of lateral stress to axial stress) is observed in figure 3 . The reduction in EPC seems to follow the same trend for all the repeatability tests $(\mathrm{pH}=2.8)$ and seems to reach a steady-state around the lower bound line depicted in the plot.

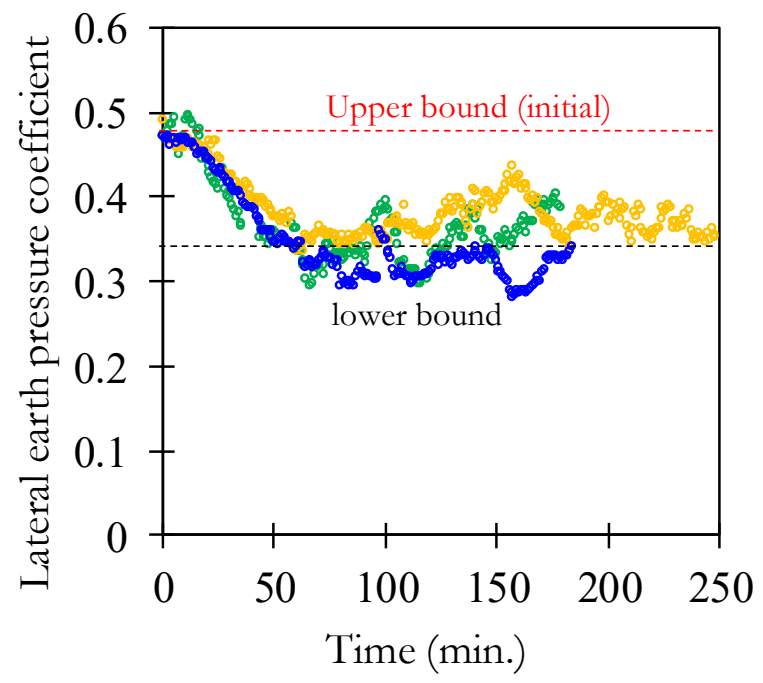

Fig. 3. EPC variation with time for $\mathrm{pH} 2.8$ (different data series correspond to independent repetitions).
The rate of dissolution controls EPC reduction. The rate of dissolution is varied by changing the inlet fluid $\mathrm{pH}$. The $\mathrm{pH}$ of 2.8 is more corrosive and brings about dissolution faster, and hence the drop of EPC is higher (figure 4) compared to $\mathrm{pH} 3.3$.

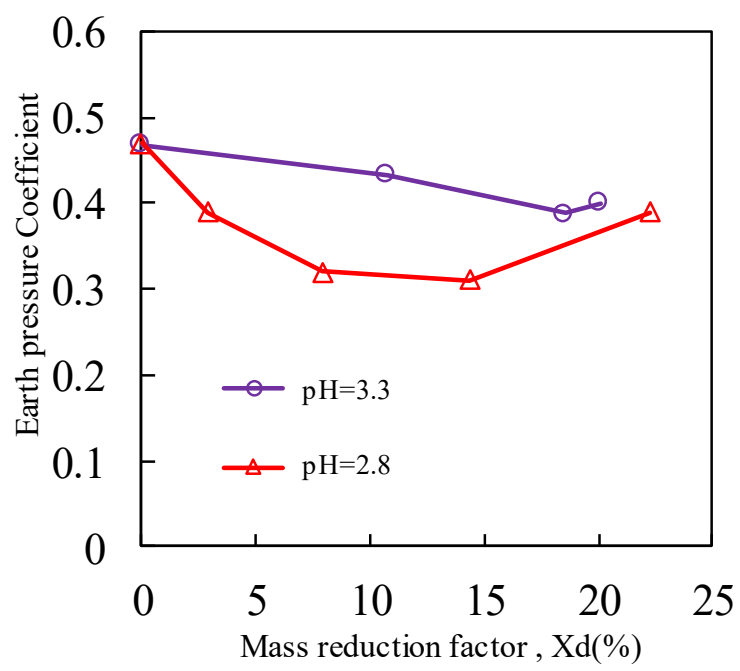

Fig. 4. EPC variation for a different rate of dissolutions.

\section{DEM study}

\subsection{Simulation details}

DEM package, namely PFC3D (Itasca, version 5), is used for the study. A cylindrical sample having dimensions the same as that of the 'soft oedometer' used in the experiments, as shown in figure 5 is used. The servo control system retains the desired axial stress on the sample through two caps which are free to move vertically. An average response within six measurement circles is taken to determine a better representation of the grain scale quantities during dissolution.

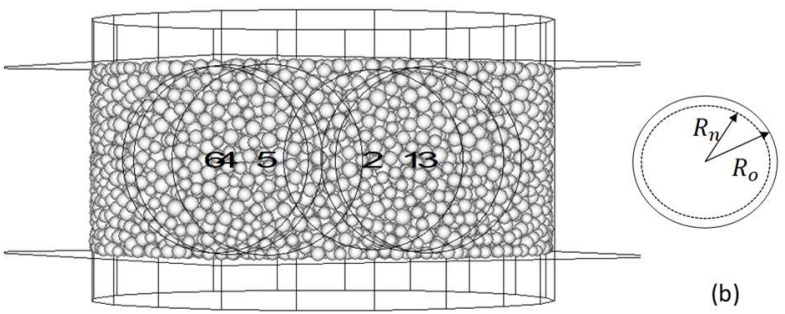

(a)

Fig. 5. (a) Diagram showing balls stacked within the boundaries. The measurement spheres have been demarcated as circles (b) descriptive diagram of radius reduction in each step.

The total number of particles utilized for the simulation is 13250 . The initial porosity of the system is kept as 0.41 . The Hertz-Mindlin contact model is utilized to capture the experimental observations. The particle size reduction is carried out by changing the incremental reduction of the radius 


$$
R_{\mathrm{xd}}=\frac{\mathrm{R}_{0}-\mathrm{R}_{\mathrm{n}}}{\mathrm{R}_{\mathrm{o}}} * 100 ;
$$

The DEM contact parameters are selected so that the resulting vertical stress-strain variation and lateral stress variation follow the same trend as the experiments in 1D compression. Figure $6(\mathrm{a} \& \mathrm{~b})$ shows the calibration of the parameters used with the experimental observations. Note that in dissolution the surface of the carbonate particles evolves, which may alter the contact properties. Therefore, to determine the stress-strain via fitting exercise is preferred over a direct measurement.
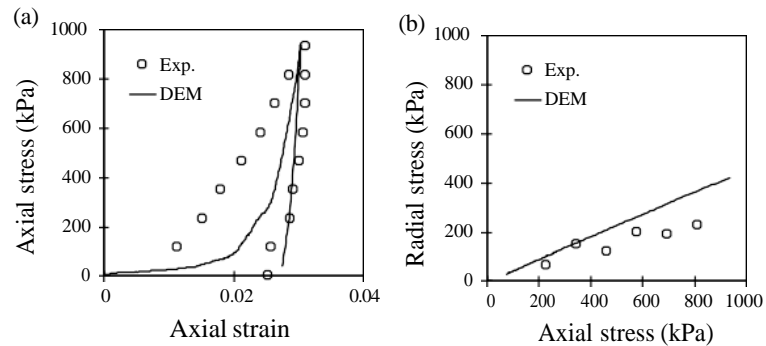

Fig. 6. Calibration of DEM parameters.

\subsection{DEM observations}

The presented DEM study offers a quantitative comparison against the experimental observation. The purpose is to mimic the lateral stress evolution trend observed in the experiments due to particle size reduction, which acts as a proxy to grain dissolution. The EPC evolution in DEM follows the same trend as the experiments, as shown in figure 7.

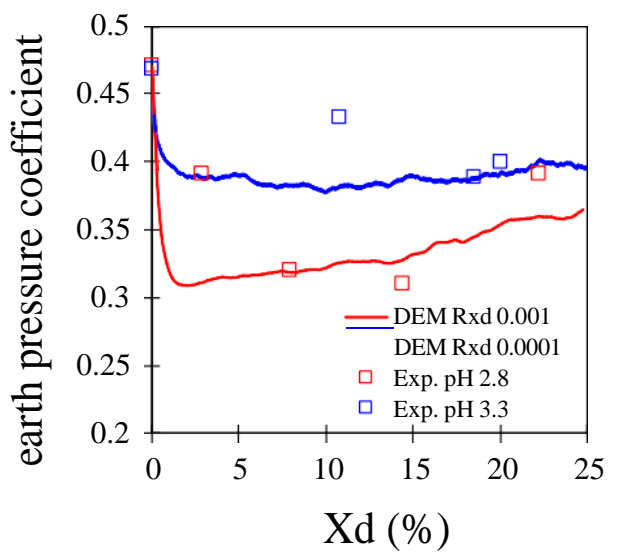

Fig. 7. Validation of EPC against concurrent chemomechanical loading response from the experiment.

Though the exact ripple nature of experimental observations is not observed, the degree of EPC reduction in DEM agrees well against the experimental counterpart. The drop in EPC is within $2 \%$ of mass reduction, showing a rapid reduction due to dissolution. The void ratio within the sample increases with dissolution irrespective of its rate, as shown in figure 7.

\section{Discussions}

The void ratio increase within the system follows a linear variation, whereas the EPC drops down the dissolution of particles are initiated. This phenomenon of EPC dropping is a result of alteration in the particle interaction. The probability distribution plot of the contact force variation during dissolution is shown in figure 8. From the figure, one can observe that the peak is shifted as dissolution proceeds. The distribution becomes narrower, indicating that the force chain is more homogeneously distributed in the granular system. A new force distribution among the particles is established as dissolution progresses, with more particles carrying less compressive force.

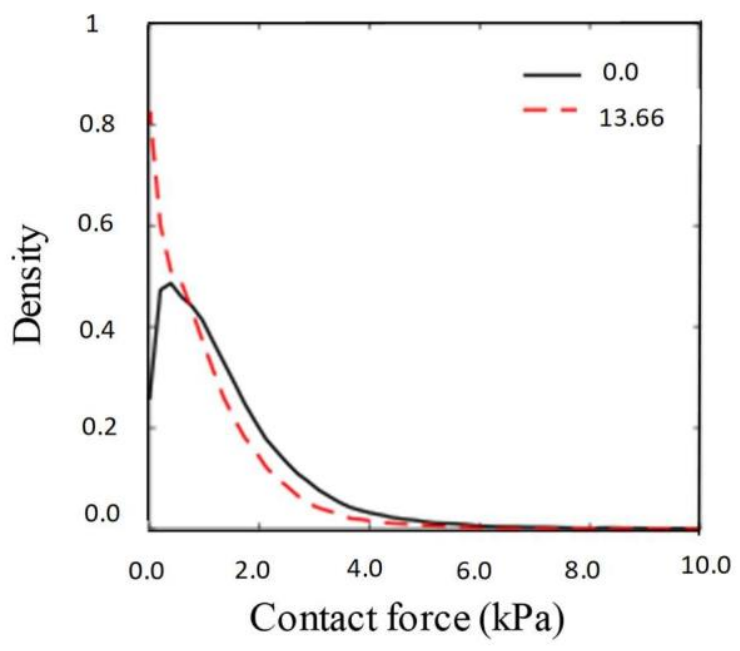

Fig. 8. Probability density of contact force at a different level of dissolution $\left(R_{\mathrm{xd}}=10^{-2}\right)$.

The microstructural evolution can be further understood from the contact information of the particles. Figure 9 shows the average coordination number variation of particles with mass dissolution. It is clear from the figure that the simulation with a higher dissolution rate had a higher reduction in coordination number. This points towards the particle rearrangement mechanism to be the guiding factor for the EPC variation. Further, the void ratio increase accompanied by particle rearrangement brings about the mechanical behaviour of the granular system undergoing dissolution.

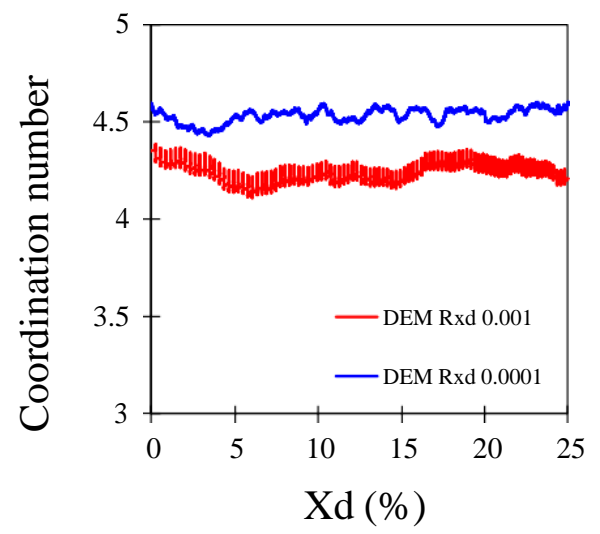

Fig. 9. Coordination number variation for two rates of dissolution.

The dissolved mass volume does not entirely add to the global void ratio due to granular rearrangement. Thus the phase relations during dissolution need further explorations to predict pure granular material [15]. 


\section{Conclusions}

Experimental study shows the reduction of EPC during chemo-mechanical loading. DEM simulations suggest that changes in coordination number during dissolution play a significant role in reducing EPC. The analyses point out that competing mechanisms between grain size reduction and grain rearrangement control the evolution of microscopic volume relations and the macroscopic stress state. Dissolution affects the homogeneous contact force distribution of a mechanically compacted granular assembly, altering the stress response.

\section{References}

[1] H. Bayesteh, T. Ghasempour, Comput. Part. Mech. 6, 327 (2019)

[2] R. Castellanza, R. Nova, J. Geotech. Geoenv. Eng. 130, 728 (2004)

[3] M. Cha, J.C. Santamarina, Géotechnique 64, 828 (2014)

[4] M.O. Ciantia, R. Castellanza, C. di Prisco, Rock Mech. Rock Eng. 48, 441 (2015)

[5] M.R. Coop, K.K. Sorensen, T. Bodas Freitas, G. Georgoutsos, Géotechnique 54, 157 (2004)

[6] I. Einav, J. Mech. Phys. Solids 55, 1274 (2007)

[7] D. Kolymbas, E. Bauer, Geotech. Test. J. 16, 263 (1993)

[8] J. McDougall, D. Kelly, D. Barreto, Acta Geotech. 8, 619 (2013)

[9] J.R. McDougall, I.C. Pyrah, Géotechnique 54, 487 (2004)

[10]H. Shin, J.C. Santamarina, J. Geotech. Geoenv. Eng. 135, 1141 (2009)

[11] B. Shipton, M.R. Coop, Soils Found. 52, 668 (2012)

[12] A. Tengattini, A. Das, A., I. Einav, Géotechnique 66, 695 (2016)

[13]Q.H. Truong, Y.H. Eom, J.S. Lee, Géotechnique 60, 293 (2010)

[14]P. Viswanath, A. Das, Effects of Particle Dissolution on the Constitutive Response of Granular Materials, in Proceedings of the $6^{\text {th }}$ Biot Conference on Poromechanics VI ASCE, 9-13 July, Paris, France (2017)

[15] P. Viswanath, A. Das, Géotechnique Lett. 10, 1 (2020) 